Bond University

Research Repository

\title{
Traits linked to executive and reward systems functioning in clients undergoing residential treatment for substance dependence
}

Lyvers, Michael; Hinton, Rachel; Gotsis, Stephanie; Roddy, Michelle; Edwards, Mark S.; Thorberg, Fred Arne

Published in:

Personality and Individual Differences

DOI:

10.1016/j.paid.2014.07.004

Licence:

CC BY-NC-ND

Link to output in Bond University research repository.

Recommended citation(APA):

Lyvers, M., Hinton, R., Gotsis, S., Roddy, M., Edwards, M. S., \& Thorberg, F. A. (2014). Traits linked to executive and reward systems functioning in clients undergoing residential treatment for substance dependence. Personality and Individual Differences, 70, 194-199. https://doi.org/10.1016/j.paid.2014.07.004

\section{General rights}

Copyright and moral rights for the publications made accessible in the public portal are retained by the authors and/or other copyright owners and it is a condition of accessing publications that users recognise and abide by the legal requirements associated with these rights.

For more information, or if you believe that this document breaches copyright, please contact the Bond University research repository coordinator. 
Traits Linked to Executive and Reward Systems Functioning in Clients Undergoing Residential Treatment for Substance Dependence 


\begin{abstract}
Traits presumed to reflect dopaminergic reward and prefrontal executive systems functioning were assessed in 100 clients undergoing residential treatment for substance dependence and a community sample of 107 social drinkers. All participants completed self-report measures of impulsivity, alexithymia, frontal systems dysfunction, sensitivity to rewards and punishments, dispositional mindfulness, alcohol use, illicit drug use, mood and demographic characteristics. The percentage of in-patients meeting the criterion for alexithymia was more than twice as high as in the community sample $(p<.0001)$. Multivariate analysis of covariance controlling for age, education, head injury and gender revealed significant differences $(p<.0001)$ between clinical and community samples such that clients scored higher on negative moods, frontal systems dysfunction, reward sensitivity, punishment sensitivity and impulsivity, and lower on dispositional mindfulness. Time in treatment was correlated only with negative mood, supporting the stability of the trait measures; controlling for negative mood eliminated group differences on punishment sensitivity and mindfulness only. Results are consistent with the notion that addiction is linked to reward sensitivity and frontal lobe deficits, with associated implications.
\end{abstract}

Keywords: addiction; alexithymia; frontal lobe 


\section{Introduction}

In recent decades biological perspectives on addiction have emphasised the intertwined roles of (1) the subcortical dopaminergic reward system as a driver of excessive substance use (Koob \& LeMoal, 1997; Volkow, Fowler \& Wang, 2004; Wise, 1998) and (2) prefrontal cortex dysfunction as the basis of the impaired self-control that characterises addictive behaviour and relapse (Lyvers, 2000; Schoenbaum \& Shaham, 2008; Spinella, 2003; Volkow \& Li, 2004). Traits presumed to reflect the functioning of these brain systems include reward sensitivity, often interpreted to index the motivational influence of the dopaminergic reward or behavioural activation system (BAS; Gray, 1987; Torrubia, Avila, Molto \& Caseras, 2001), and traits such as impulsivity, disinhibition, executive deficit and alexithymia (difficulties with identifying and describing one's emotions), which have been linked to abnormalities of prefrontal cortex function as well as risky or problematic substance use (Berthoz et al., 2002; Chen et al., 2007; Dawe, Gullo \& Loxton, 2004; Lyvers, Duff, Basch \& Edwards, 2012; Pinard, Negrete, Annable, \& Audet, 1996; Spinella, 2004; Thorberg et al., 2009). A combination of inherently high reward sensitivity and deficient executive self-regulation appears to be significantly associated with risky substance use and addiction (Dawe \& Loxton, 2004; Dolan, Bechara, \& Nathan, 2008; Ivanov, Schulz, London \& Newcorn, 2008; Lyvers, Carlopio, Bothma \& Edwards, 2013; Lyvers, Jamieson \& Thorberg, 2013). Recent attention has also focused on the possible role of mindfulness as a protective factor against addiction and relapse (Bowen \& Vieten, 2012). Mindfulness, defined as "awareness and attention to present events and experiences" (Brown \& Ryan, 2003, p. 824), appears to reflect higher-order, meta-cognitive functioning (Chambers et al., 2009) and is inversely related to impulsivity, alexithymia, and indices of frontal systems dysfunction (Lyvers, Makin, Toms, Thorberg \& Samios, in press). Mindfulness thus appears to be another aspect of executive cognition. 
Based on the notion that addiction is associated with inherently high BAS responsiveness as well as deficiencies in aspects of prefrontal cortex function such as impulse control, mood regulation, and executive cognition (Dawe \& Loxton, 2004; Dolan et al., 2008; Ivanov et al., 2008), the present study examined self-report indices of reward sensitivity, frontal systems dysfunction, impulsivity, alexithymia, and mindfulness in abstinent, detoxified clients undergoing in-patient treatment for substance dependence and in a comparison sample of non-intoxicated social drinkers from the local community. These measures had previously shown significant correlations with risky substance use in nonclinical community samples for a variety of substances (Lyvers, Carlopio et al., 2013; Lyvers, Jamieson et al., 2013; Lyvers et al., 2012; Pinard et al., 1996) but had not previously been examined together in a clinical sample of substance dependent clients. Other variables that were previously found to influence responding on at least some of the measures of interest to the present investigation were also assessed in the present study and their influence controlled via covariate analysis. The clinical sample was expected to score higher than the community sample on measures of negative moods, especially among those early in treatment; but independent of transient mood differences, indices of more stable traits reflecting inherent executive dysregulation (prefrontal cortex) and reward sensitivity (BAS) were expected to be significantly elevated in the addicts, in line with theoretical expectations.

\section{Method}

\subsection{Participants}

Substance dependent clients from three Therapeutic Community (TC) addiction treatment centres in southeastern Queensland, Australia, were recruited for the present study over a 10 week period. A $\$ 40$ gift voucher was offered as an incentive for participation. Abstinence was confirmed by random urine testing. Residential treatment programs were for a minimum of six months. All client participants were self-referred, had completed more than 
14 days in treatment to rule out an influence of detoxification or acute withdrawal on responses, and were not diagnosed with a comorbid neurological or psychological disorder (these were stated criteria for participation). The final clinical sample consisted of $100 \mathrm{TC}$ inpatients undergoing treatment for substance (alcohol and/or illicit drug) dependence (58 males, 42 females; age range 18-63 years, $M=32.47, S D=11.26$ ) who on average had been in treatment for 108 days with a range of 17-612 days. The community sample was recruited through advertisements in a local newspaper over the same 10 week period. The advertisements asked for social drinkers who were not currently undergoing treatment for any neurological or psychological disorder. A $\$ 40$ gift voucher was specified as an incentive for participation. Interested community members were instructed to attend the university campus where the testing would occur at a pre-arranged time and place. The final community sample consisted of 107 social drinker volunteers from the local southeast Queensland community (43 males, 64 females; age range $18-48$ years, $M=26.65$, $S D=6.50$ ). Volunteers from either group were excluded from the final sample if their responses on a demographics and screening questionnaire indicated a current neurological condition (2 addict volunteers). In addition community participants were excluded if their questionnaire responses or alcohol breath test indicated recent intoxication (5 volunteers); if their scores on the Alcohol Use Disorders Identification Test (AUDIT; Babor, de la Fuente, Saunders \& Grant, 1992) or the Drug Use Disorders Identification Test (DUDIT; Berman, Bergman, Palmstierna \& Schlyter, 2003 ) indicated likely substance dependence (6 volunteers); or if their demographic questionnaire responses indicated previous treatment for a substance problem (1 volunteer). The final samples described above also reflect the exclusion of 8 multivariate outliers identified via Mahalanobis Distance using boxplots. No participants in either sample were of Aboriginal background. 
In the clinical sample $21 \%$ indicated that alcohol was their primary problem, $11 \%$ indicated stimulants (cocaine or amphetamines), 5\% indicated cannabis and 3\% indicated opiates such as heroin. The remainder $(60 \%)$ indicated that their problem involved multiple substances, generally combinations of cannabis, stimulants and/or opiates with alcohol. All participants provided informed consent prior to participation and could withdraw from the study at any time without penalty. Participants were instructed to not provide identifying information on their questionnaires in order to ensure anonymity. The research protocol was approved by the university ethics committee and treatment centres prior to data collection.

\subsection{Procedure}

Clients were recruited to complete the pen and paper survey by a staff member at the addiction treatment facility. They were asked to place the completed survey in a provided envelope and seal it when finished. The staff member then collected the sealed envelopes and passed them on to the researchers. Community participants completed the same survey in a designated testing room at a local university. They were asked to place the completed survey in a provided envelope, seal it and hand it to one of the researchers when finished. Survey completion required approximately $30-45$ minutes.

\subsection{Materials}

The following questionnaires were administered to all participants.

2.3.1. A Demographics questionnaire asked for participant age, gender, age at onset of drinking/drug use, years of education, neurological status, current or previous treatment for a substance problem or other psychological disorder, number of days in treatment (clinical sample only), whether they had ever suffered a serious head injury, and recent alcohol or drug use.

2.3.2. The Depression Anxiety Stress Scales -21 (DASS-21; Lovibond \& Lovibond, 1995 ) is a 21-item self-report questionnaire consisting of three scales (each with 7 items) 
designed to measure the emotional states of depression (e.g., "I felt down-hearted and blue"), anxiety (e.g., "I felt scared without any good reason"), and stress (e.g., "I found it hard to wind down") upon reflection of the prior week. Items are rated on a 4-point Likert scale, ranging from $0=$ did not apply to me at all, to $3=$ applied to me very much or most of the time. Items are summed within each scale, with higher scores indicating higher levels of depression, anxiety, and stress. In the present study Cronbach's alpha for the overall DASS21 was .96 .

\subsubsection{The Sensitivity to Punishment and Sensitivity to Reward Questionnaire} (SPSRQ; Torrubia et al., 2001) is comprised of two subscales, Sensitivity to Punishment (SP) and Sensitivity to Reward (SR). Each subscale has 24 items measuring an individual's motivational propensity to engage in either approach (SR) or avoidance (SP) behaviour in the presence of either rewarding or punishing stimuli, respectively. Items are answered via dichotomous yes (1) or no (0) responses, which are summed for each subscale. The SPSRQ is based on Gray's (1987) Reinforcement Sensitivity Theory (RST), such that the SR scale was designed to evaluate the Behavioural Activation System (BAS) and the SP scale was designed to evaluate the Behavioural Inhibition System (BIS). The SPSRQ has been established as a valid measure of the BAS and BIS components of RST (Caseras, Avila, \& Torrubia, 2003; Loxton \& Dawe, 2001). In the present study Cronbach's alpha was .81 for the SR scale and .85 for SP.

2.3.4. The Mindful Attention Awareness Scale (MAAS; Brown \& Ryan, 2003) is a 15item self-report measure of dispositional mindfulness. Items are negatively worded as Brown and Ryan (2003) felt statements reflecting states of "mindlessness" were "more accessible to most individuals, given that mindless states are much more common than mindful states" (p. 826). Answers are given on a 6-point Likert scale, where $1=$ almost always and $6=$ almost never. Questions ask about awareness and attention, e.g., "I find it difficult to stay focused on 
what's happening in the present," "I do jobs or tasks automatically, without being aware of what I'm doing." Higher scores indicate higher trait mindfulness. A single factor solution was yielded from a sample of 727 university students (MacKillop \& Anderson, 2007). MAAS scores have shown a positive association with prefrontal cortex activation and a negative relationship with amygdala activation (Creswell et al., 2007), in line with theoretical expectations. In the present study Cronbach's alpha for the MAAS was .91.

2.3.5. The Frontal Systems Behavior Scale (FrSBe; Grace \& Malloy, 2001) is a 46item questionnaire designed to measure neurobehavioral traits related to dysfunction in three key prefrontal systems: the anterior cingulate (Apathy subscale, 14 items; e.g., 'Sit around doing nothing'), orbitofrontal cortex (Disinhibition subscale, 15 items; e.g., "Do or say embarrassing things"), and dorsolateral prefrontal cortex (Executive Dysfunction subscale, 17 items; e.g., "Repeat certain actions or get stuck on certain ideas"). For brain injured patients the FrSBe is used to assess changes in behaviour by obtaining pre- and post-lesion ratings on a 5 -point Likert scale $(1=$ almost never, $2=$ seldom, $3=$ sometimes, $4=$ frequently, $5=$ almost always), however in the present study only self-ratings of current behavioral functioning were obtained as in previous work conducted on non-brain-injured samples (e.g., Lyvers et al, 2012; Spinella, 2003; Stout et al., 2003). The first 32 items represent deficits and are positively phrased, whereas the last 14 items are negatively phrased and reverse scored. Items are summed with higher ratings indicating higher levels of impairment for the subscales as well as the total score. In the present study Cronbach's alpha for the total FrSBe was .92 .

2.3.6. The Barratt Impulsiveness Scale (BIS-11; Patton et al., 1995) is a 30-item selfreport scale designed to measure trait impulsivity. Answers are given on a 4-point Likert scale $(1=$ rarely/never to $4=$ almost always/always $)$ to questions reflecting cognitive instability and inattention (8 items, e.g., "I don’t pay attention”), motor impulsiveness (11 
items, e.g., "I do things without thinking"), and non-planning impulsivity (11 items, e.g., "I plan tasks carefully," reverse scored). Items are summed to produce an overall score, where higher scores indicate greater impulsiveness. Total scores can range from 30 to 120. Convergent validity was established with neuropsychological measures that have shown sensitivity to prefrontal cortex dysfunction (Spinella, 2004). In the present study Cronbach's alpha for the total BIS-11 was .81.

2.3.7. The Toronto Alexithymia Scale-20 (TAS-20; Bagby, Parker \& Taylor, 1994) is a 20-item self-report questionnaire used to assess trait levels of alexithymia. Items are rated on a 5-point Likert scale $(1=$ strongly agree to $5=$ strongly disagree $)$ and summed within each subscale and then overall to create total scores that range from 20 to 100 , with higher scores indicating greater levels of alexithymia. Cut-off scores have been empirically derived for the TAS-20, whereby $>61=$ alexithymia and $<51=$ no alexithymia (Bagby, Taylor $\&$ Parker, 1994). The TAS-20 has sound validity and reliability (Thorberg et al., 2010) as well as a confirmed three factor structure supported in clinical (Swift, Stephenson, \& Royce, 2006) as well as non-clinical (Parker, Taylor, \& Bagby, 2003) samples: difficulty identifying feelings (DIF; e.g., "I have feelings that I can't quite identify"), difficulty describing feelings (DDF; e.g., "People tell me to describe my feelings more"), and externally-oriented thinking (EOT; e.g., "I feel close to someone, even in moments of silence," reverse-scored). In the present study Cronbach's alpha for the TAS-20 was .78.

2.3.8. The Alcohol Use Disorders Identification Test (AUDIT; Babor et al., 1992) is a 10 item self-report survey used to identify low-risk, hazardous, harmful and dependent drinking in adolescents and adults. Response options are based on a Likert-type scale ranging from 0 to 4. Three items measure alcohol consumption, e.g., "In the past week, how often did you have a drink containing alcohol?" Three items assess alcohol dependence, e.g., "How often, during the past year, have you found that you were not able to stop drinking once you 
had started?" Four items evaluate alcohol-related harm, e.g., "How often during the past year have you had a feeling of guilt or remorse after drinking?" Scores are calculated by summation of all 10 items and can range between 0 and 40. Scores between 0 and 7 are considered "Low Risk," a score between 8 and 15 suggests "Hazardous" drinking and a score of 16 or above indicates "Harmful" drinking. A score of 4 or higher on the combined dependence items suggests likely alcohol dependence, as does a total score of 20 or higher (Babor et al., 1992). AUDIT was used in the present study to identify and remove any community participants with likely alcohol dependence. TC clients were instructed to base their AUDIT responses on their drinking behaviour prior to admission into treatment. In the present study Cronbach's alpha for the AUDIT was .95.

2.3.9. The Drug Use Disorders Identification Test (DUDIT; Berman et al., 2003) was developed as a parallel measure to the AUDIT for drugs other than alcohol, provides a list of such drugs, and has similar items and scoring procedures to the AUDIT. Total scores can range from 0 to 44 . A total score of 25 or higher suggests likely drug dependence (Berman et al., 2003). DUDIT was used in the present study to identify and remove any community participants with likely drug dependence. TC clients were instructed to base their DUDIT responses on their drug using behaviour prior to admission into treatment. In the present study Cronbach's alpha for the DUDIT was .98.

\section{Results}

3.1. Alexithymia. Clinical status was significantly associated with alexithymia as defined by TAS-20 cut-off, $\chi^{2}(2)=15.92, p<.0001$. Of the clinical sample of 100 inpatients, 40 (i.e., $40 \%$ ) were defined as alexithymic by TAS-20 compared to only 20 out of 107 (i.e., $18.69 \%$ ) of the community sample. Alexithymia was not associated with gender overall, $p=.99$. 
3.2. Treatment Duration. In the clinical sample, number of days in TC treatment ranged from 17 to 612 days $(M=108$ days $)$ and was examined using Pearson correlations in relation to the questionnaire measures. As expected the only variables that were significantly correlated with number of days in treatment were the DASS negative mood scales: Depression, $r(100)=-.27, p=.006$; Anxiety, $r(100)=-.28, p=.004$; and Stress, $r(100)=$ $-.28, p=.005$. Depression, anxiety and stress decreased as a function of days in treatment. No other variables - neither BIS-11, TAS-20, FrSBe scales, SR, SP nor MAAS - correlated with days in treatment (all $p>.20$ ), suggesting that these indexed relatively stable traits in this sample.

3.3. Intercorrelations. Intercorrelations for the entire sample are shown in Table 1. As can be seen in the table, each trait measure was significantly correlated with AUDIT and DUDIT scores as well as the DASS mood scales and most trait measures. The same pattern of correlations was apparent across samples and genders.

3.4. Group Comparisons. Clinical and community samples significantly differed on demographic variables, hence the potential influence of these was controlled via covariate analysis. There were relatively more men in the clinical sample (58\%) than in the community sample $(40 \%), \chi^{2}(1)=6.57, p=.01$. The clinical sample tended to be older $(M=32.47$ years, $S D=11.26)$ than the community sample $(M=26.65$ years, $S D=6.50), F(1,205)=21.05, p<$ .0001 . The community sample had completed significantly more years of education $(M=$ 14.03 years, $S D=2.88)$ than the clinical sample $(M=11.02$ years, $S D=2.73), F(1,205)=$ 59.43, $p<.0001$. More participants in the clinical sample reported ever having suffered a serious head injury (29\%) than in the community sample $(2 \%), \chi^{2}(1)=29.88, p<.0001$. Thus age, education level and past head injury were included as covariates in a 2 (clinical vs. community) X 2 (male vs. female) multivariate analysis of covariance (MANCOVA) on the DASS scales, FrSBe scales, SPSRQ scales, MAAS, and BIS-11. Levene's Test was 
significant $(p<.05)$ for DASS Depression and Anxiety as well as BIS-11, so the analysis was conducted again using square root transformations for Depression and Anxiety and a log 10 transformation for BIS-11; this reduced (BIS-11) or eliminated (Depression, Anxiety) the significant Levene's Test results for these variables without altering the results of the MANCOVA, hence MANCOVA results for untransformed variables are reported here.

The overall multivariate effect of sample (clinical vs. community) was significant according to Pillai's Trace, $F(7,194)=16.16, p<.0001, \eta 2=.37$, observed power $=1$. Univariate differences were significant at $p<.0001$ for all dependent measures with $\eta 2$ ranging from .07 to .30 indicating medium to large effect sizes. Table 2 shows that, compared to the community sample, the clinical sample had higher levels of depression, anxiety, stress, impulsivity, reward sensitivity, punishment sensitivity, disinhibition, apathy, and executive dysfunction, and lower levels of dispositional mindfulness. This was after controlling for the contributions of age, gender, education level and past serious head injury status to group differences. The overall sample $\mathrm{X}$ gender interaction was not significant, $F(7,194)=1.27, p$ $=.27$. However the overall multivariate effect of gender was significant, $F(7,194)=3.80, p=$ .001 , with a univariate difference for SP, $F(1,200)=13.01, p<.0001$. Consistent with previous work (e.g., Lyvers et al., 2012), women scored higher on SP overall $(M=12.51, S D$ $=5.24)$ than men $(M=10.66, S D=5.67)$.

Table 2 also shows group results for AUDIT and DUDIT. As expected, responses of clients on both measures were consistent with substance dependence (see Table 2), whereas for the community sample DUDIT scores averaged slightly above 0 with a range of 0 to 16 , and AUDIT scores averaged just above the cut-off between Low Risk and Hazardous Drinking and ranged from 1 to 18 .

Given that DASS scores declined in relation to time in treatment in the clinical sample as described above, the MANCOVA was conducted a second time with a composite DASS 
score as an additional covariate to see if any of the above group differences changed after controlling for transient negative mood. Group differences remained significant at the multivariate $(p<.0001)$ and univariate levels $(p<.05$ to $p<.0001)$ in this analysis with the exceptions of SP $(p=.17)$ and MAAS $(p=.26)$ which were highly correlated with the DASS scales (see Table 1).

\section{Discussion}

As expected the percentage of addicts defined as alexithymic by TAS-20 cut-off was more than twice as high as in the community sample, consistent with previous work showing associations between alexithymia and risky or problematic substance use (Lyvers, Carlopio et al., 2013; Lyvers, Jamieson et al., 2013; Pinard et al., 2008; Thorberg et al., 2009). The other trait measures also differed in expected ways between samples, consistent with the notion that addiction is associated with elevated reward sensitivity and deficient executive self-regulation as mediated by corresponding brain systems (BAS and prefrontal cortex respectively) (Dawe et al., 2004; Dolan et al., 2008; Ivanov et al., 2008; Lyvers et al., 2012). Self-reported levels of negative mood were lower in clients who had been in addiction treatment longer, but the primary trait indices of interest still showed significant group differences even after controlling for mood - with the exceptions of punishment sensitivity and mindfulness, which were highly correlated with the DASS mood index. Indices of impulsivity, reward sensitivity, apathy, disinhibition and executive dysfunction all remained significantly higher in the clinical sample than in the community sample even after controlling for negative moods, in addition to controlling for potential contributions of differences in age, education, head injury and gender to group differences, and did not vary in relation to duration of treatment (which ranged from 17 to 612 days) in the clinical sample.

\subsection{Conclusions}

Indices of traits presumed to reflect the intertwined functioning of the subcortical 
dopaminergic reward and prefrontal cortical executive systems showed expected differences between in-patient and community samples that are likely to have predated substance use to a significant extent - though a sustained impact of addictive drugs on the brain is a competing account that cannot be excluded (Crews, He \& Hodge, 2007; Lyvers, 2000; Volkow et al., 2004). Irrespective of the basis of such differences, the present findings do reinforce a need to identify and target client characteristics that may be likely to promote addiction and relapse, such as alexithymia, impulsivity and executive dysfunction, in addition to dealing with negative mood issues during treatment for substance dependence. For example, TC client training in skills such as executive self-monitoring, emotional awareness and impulse control may prove beneficial (Alfonso et al., 2011; Thorberg et al., 2009). The present findings also point to the need for longitudinal studies to disentangle the roles of pre-drug personality versus drug use history to client characteristics associated with addiction, as well as the related mechanisms of change in recovery. 


\section{References}

Alfonso, J. P., Caracuel, A., Delgado-Pastor, L., \& Verdejo-García, A. (2011). Combined goal management training and mindfulness meditation improve executive functions and decision-making performance in abstinent polysubstance abusers. Drug \& Alcohol Dependence, $117,78-81$.

Babor, T.F., de la Fuente, J.R., Saunders, J. \& Grant, M. (1992). AUDIT: Alcohol Use Disorders Identification Test. Geneva: World Health Organization.

Bagby, R.M., Parker, J.D., \& Taylor, G.J. (1994). The twenty-item Toronto Alexithymia Scale: Item selection and cross-validation of the factor structure. Journal of Psychosomatic Research, 38, 23-32.

Bagby, R., Taylor, G., \& Parker, J. (1994). The twenty-item Toronto Alexithymia Scale: II Convergent, discriminant, and concurrent validity. Journal of Psychosomatic Research, 38, 33-40.

Berman, A.H., Bergman, H., Palmstierna, T., \& Schlyter, F. (2003). Drug Use Disorders Identification Test (DUDIT) manual. Stockholm: Karolinska Institute.

Berthoz, S., Artiges, E., Van de Moortele, P. F., Poline, J.-B.,Rouquette, S., \& Consoli, S. M. (2002). Effect of impaired recognition and expression of emotions on frontocingulate cortices: An fMRI study of men with alexithymia. American Journal of Psychiatry, 159, 961-967.

Bowen, S. \& Vieten, C. (2012). A compassionate approach to the treatment of addictive behaviors: The contributions of Alan Marlatt to the field of mindfulness-based interventions. Addiction Research and Theory, 20, 243-249.

Brown, K.W., \& Ryan, R.M. (2003). The benefits of being present: Mindfulness and its role in psychological well-being. Journal of Personality \& Social Psychology, 84, 822848. 
Caseras, X., Avila, C., \& Torrubia, R. (2003). The measurement of individual differences in behavioural inhibition and behavioural activation systems: A comparison of personality scales. Personality \& Individual Differences, 34, 999-1013.

Chambers, R., Gullone, E., \& Allen, N.B. (2009). Mindful emotion regulation: An integrative review. Clinical Psychology Review, 29, 560-572.

Chen, A. C. H., Porjesz, B., Rangaswamy, M., Kamarajan, C., Tang, Y., Jones, K. A., et al. (2007). Reduced frontal lobe activity in subjects with high impulsivity and alcoholism. Alcoholism: Clinical and Experimental Research, 31(1), 156-165.

Creswell, J., Way, B.M., Eisenberger, N.I., \& Lieberman, M.D. (2007). Neural correlates of dispositional mindfulness during affect labeling. Psychosomatic Medicine, 69, 560565.

Crews, F., He, J., \& Hodge, C. (2007). Adolescent cortical development: A critical period of vulnerability for addiction. Pharmacology Biochemistry and Behavior, 86, 189-199.

Dawe, S., Gullo, M., J., \& Loxton, N. J. (2004). Reward drive and rash impulsiveness as dimensions of impulsivity: Implications for substance misuse. Addictive Behaviors, 29, 1389-1405.

Dawe, S. \& Loxton, N. J. (2004). The role of impulsivity in the development of substance use and eating disorders. Neuroscience and Biobehavioural Reviews, 28, 343-351.

Dolan, S.L., Bechara, A., \& Nathan, P.E. (2008). Executive dysfunction as a risk marker for substance abuse: The role of impulsive personality traits. Behavioral Sciences and the Law, 26, 799-822.

Grace, J. \& Malloy, P. F. (2001). Frontal Systems Behavioral Scale (FrSBe): Professional manual. Lutz, F. L.: Psychological Assessment Resources.

Gray, J.A. (1987). The psychology of fear and stress. Cambridge: Cambridge University Press. 
Ivanov, I., Schulz, K. P., London, E. D., \& Newcorn, J. H. (2008). Inhibitory control deficits in childhood and risk for substance use disorders: A review. American Journal of Drug and Alcohol Abuse, 34, 239-58.

Koob G.F,„\& Le Moal, M. (1997). Drug abuse: Hedonic homeostatic dysregulation. Science, 278, 52-58.

Lovibond, S.H., \& Lovibond, P.F. (1995). Manual for the Depression Anxiety and Stress Scales. Sydney, Australia: Psychological Foundation.

Loxton, N. J., \& Dawe, S. (2001). Alcohol abuse and dysfunctional eating in adolescent girls: The influence of individual differences in sensitivity to reward and punishment. International Journal of Eating Disorders, 29, 455-462.

Lyvers, M. (2000). "Loss of control" in alcoholism and drug addiction: A neuroscientific interpretation. Experimental and Clinical Psychopharmacology, 8, 225-249.

Lyvers, M., Carlopio, C., Bothma, V., \& Edwards, M. S. (2013). Mood, mood regulation expectancies and frontal systems functioning in current smokers versus neversmokers in China and Australia. Addictive Behaviors, 38, 2741-2750.

Lyvers, M., Duff, H., Basch, V., \& Edwards, M. (2012). Influences of rash impulsiveness and reward sensitivity on risky drinking in university students: Evidence of mediation by frontal systems. Addictive Behaviors, 37, 940-946.

Lyvers, M., Jamieson, R., \& Thorberg, F.A. (2013). Risky cannabis use is associated with alexithymia, frontal lobe dysfunction and impulsivity in young adult cannabis users. Journal of Psychoactive Drugs, 45, 394-403.

Lyvers, M., Makin, C., Toms, E., Thorberg, F.A., \& Samios, C. Trait mindfulness in relation to alexithymia, emotion regulation, mood and everyday executive functioning. Mindfulness, in press. Early online: http://link.springer.com/article/10.1007/s12671013-0213-y 
MacKillop, J., \& Anderson, E. J. (2007). Further psychometric validation of the Mindful Attention Awareness Scale (MAAS). Journal of Psychopathology \& Behavioral Assessment, 29, 289-293.

Parker, J.D.A., Taylor, G.J., \& Bagby, R.M. (2003). The 20-Item Toronto Alexithymia Scale III: Reliability and factorial validity in a community population. Journal of Psychosomatic Research, 55, 269-275.

Patton, J.H., Stanford, M.S., \& Barratt, E.S. (1995). Factor structure of the Barratt Impulsiveness Scale. Journal of Clinical Psychology, 51, 768-774.

Pinard, L., Negrete, J.C., Lawrence, A., \& Audet, N. (1996). Alexithymia in substance abusers: Persistence and correlates of variance. American Journal on Addictions, 5, 32-39.

Schoenbaum, G., \& Shaham, Y. (2008). The role of the orbitofrontal cortex in drug addiction: A review of preclinical studies. Biological Psychiatry, 63, 256-262.

Spinella, M. (2003). Relationship between drug use and prefrontal associated traits. Addiction Biology, 8, 67-74.

Spinella, M. (2004). Neurobehavioral correlates of impulsivity: Evidence of prefrontal involvement. International Journal of Neuroscience, 114, 95-104.

Stout, J.C., Ready, R.E., Grace, J., Malloy, P.F., \& Paulsen, J.S. (2003). Factor analysis of the Frontal Systems Behaviour Scale (FrSBe). Assessment, 10, 79-85.

Swift, L., Stephenson, R., \& Royce, J. (2006). The 20-item Toronto Alexithymia Scale: Validation of factor solutions using confirmatory factor analysis on physiotherapy outpatients. Psychology \& Psychotherapy, 79, 83-88.

Thorberg, F.A., Young, R., Sullivan, K.A., \& Lyvers, M. (2009). Alexithymia and alcohol misuse disorders: A critical review. Addictive Behaviors, 34, 237-245. 
Thorberg, F. A., Young, R. M., Sullivan, K. A., Lyvers, M., Connor, J. P., \& Feeney, G. F. X. (2010). A psychometric comparison of the Toronto Alexithymia Scale (TAS-20) and the Observer Alexithymia Scale (OAS) in an alcohol-dependent sample. Personality and Individual Differences, 49(2), 119-123.

Torrubia, R., Avila, C., Molto, J., \& Caseras, X. (2001). The Sensitivity to Punishment and Sensitivity to Reward Questionnaire (SRSPQ) as a measure of Gray's anxiety and impulsivity dimensions. Personality and Individual Differences, 31, 837-862.

Volkow, N.D., \& Li, T.K. (2004). Drug addiction: The neurobiology of behaviour gone awry. Nature Reviews Neuroscience, 5, 963-970.

Volkow, N.D., Fowler, J.S., \& Wang, G.J. (2004). The addicted human brain viewed in the light of imaging studies: Brain circuits and treatment strategies. Neuropharmacology, 47, 3-13.

Wise, R.A. (1998). Drug-activation of brain reward pathways. Drug \& Alcohol Dependence, $51,13-22$. 